\title{
Cigarrillos eléctrónicos. ¿Podemos recomendar su uso?
}

\author{
GONZALO ALVEAR T.*, LUIS SANTIBÁÑEZ S.*, \\ VÍCTOR RAMÍREZ S.* y RICARDO SEPÚLVEDA M.*
}

\section{Electronic cigarettes. Can we recommend its use?}

Electronic cigarettes (Cig-e) are devices that contain a liquid composed of a solvent, flavors and, in most cases, nicotine. This liquid, when heated in the device, vaporizes, being aspirated by the user. In recent years, the use of Cig-e has increased rapidly, especially among schoolchildren and young people, with no clear evidence of safety and long-term effects to date. In this article we review the existing evidence regarding the effects and safety of each of the components of Cig-e and analyze their potential indications in different clinical scenarios.

Key words: Electronic cigarettes; nicotine, flavoring agents; nebulizers and vaporizers; solvents.

\section{Resumen}

Los cigarrillos electrónicos (Cig-e) son dispositivos que contienen un líquido compuesto por un solvente, saborizantes y, en la mayoría de los casos, nicotina. Este líquido, al ser calentado en el dispositivo, se vaporiza, siendo aspirado por el usuario. En los últimos años, el uso de los Cig-e ha aumentado rápidamente, especialmente entre escolares y gente joven, sin existir hasta el día de hoy evidencias ciertas sobre su seguridad y efectos a largo plazo. En este artículo revisamos la evidencia existente en cuanto a los efectos y seguridad de cada uno de los componentes de los Cig-e y analizamos sus potenciales indicaciones en distintos escenarios clínicos.

Palabras clave: Cigarrillos electrónicos, nicotina, saborizantes, nebulizadores y vaporizadores, solventes.

\section{Introducción}

Los cigarrillos electrónicos (Cig-e) son dispositivos que contienen un líquido que, al ser calentado, producen un aerosol (vapor) que es inhalado ("vapeado") por el usuario. Los Cig-e tienen cuatro partes: la batería, el elemento calentador, la cámara de vaporización y el cartucho de la solución, que es el que contiene el líquido que se convierte en el aerosol. El líquido contiene, en general, un solvente, que puede ser glicerina vegetal, propilenglicol o una mezcla de ellos, y uno o más saborizantes. La nicotina, si bien está presente en la mayoría de los líquidos de los Cige, no es requisito. El proceso de generación del vapor del Cig-e es completamente diferente al del humo del cigarrillo, el que es generado por com- bustión. En el Cig-e, la activación del mecanismo de calentamiento se realiza gracias a un sensor que detecta cuando el usuario realiza un puff o "vapeo", el que activa una bobina electrónica que calienta y vaporiza el líquido, creando un humo de vapor visible ${ }^{1-4}$.

En los últimos años, el uso de los Cig-e ha aumentado rápidamente, especialmente entre los escolares y la gente joven ${ }^{5.6}$, habiéndose más que triplicado en adolescentes desde el año 2011 y duplicado entre el 2013 y 2014 en los adultos jóvenes ${ }^{7}$, sobrepasando al cigarrillo convencional el año 2014 en EE.UU.? . Existe en la actualidad un gran debate en cuanto a la seguridad y efectos sobre la salud con su uso a largo plazo, tanto del usuario como de los que están expuestos a este vapor, además de su potencial de adicción,

* Grupo Respiratorio Integramédica. Américo Vespucio 1501. Cerrillos. Región Metropolitana, Chile. 
el eventual traspaso al uso del cigarrillo convencional ${ }^{8,9-11}$. Frente a estos aspectos, existen posiciones diametralmente opuestas entre distintas organizaciones internacionales, algunas con posiciones absolutamente restrictivas y otras más permisivas. Entre estas últimas están las autoridades de salud pública inglesa, que sugieren que los Cig-e son un $95 \%$ más seguros que los cigarrillos convencionales ${ }^{12}$ ya que no contienen, o se encuentran en niveles mucho más bajos, los constituyentes del humo del cigarrillo convencional responsables de los efectos adversos sobre la salud. Esto, principalmente porque los productos derivados de la combustión de los cigarrillos convencionales, más que el tabaco o la nicotina en sí mismos, son la principal causa de los efectos dañinos sobre la salud ${ }^{13} \mathrm{y}$, los Cig-e, sólo calientan un líquido para aerosolizarlo, sin combustión. Además, los principales químicos presentes en el vapor de los Cig-e no se han asociado aún a ningún riesgo serio sobre la salud ${ }^{14}$. Esta posición más permisiva la comparten el Royal College of Physicians of London ${ }^{15}$ y la Action on Smoking Health (ASH) del Reino Unido ${ }^{16}$.

Por el lado más restrictivo están la agencia reguladora europea y la FDA (Food and Drug Administration, EE.UU.), las que, debido a los potenciales efectos adversos de estos dispositivos sobre la salud, los han calificado como productos de tabaco ${ }^{17,18}$, es decir, productos que podrían producir los mismos, o similares efectos sobre la salud, que los cigarrillos convencionales. Esta posición de restricción la comparten también la Organización Mundial de la Salud: $\mathrm{OMS}^{19}$, la Comisión Europea ${ }^{20}$, la British Medical Association, Centers for Disease Control and Prevention de EE.UU. y la American Lung Association ${ }^{21}$.

Independientemente de estas posiciones y debido a este ambiente de incertidumbre sobre los efectos de los Cig-e, el objetivo de esta revisión es analizar la evidencia disponible hasta la fecha sobre los potenciales efectos del Cig-e sobre la salud respiratoria de los consumidores con el fin de responder la pregunta: ¿podemos recomendar el uso de los Cig-e?

\section{Efectos del uso del Cig-e: Evidencias}

La determinación de los efectos reales del Cige sobre la salud de los consumidores depende de varios factores: a) tipo del dispositivo utilizado; b) contenido del líquido y del aerosol producido; c) el comportamiento y experiencia del usuario; d) traslado de los efectos encontrados en estudios in vitro y en animales al hombre.

\section{a) Tipo de dispositivo utilizado}

Existe hoy en día una multitud de dispositivos de Cig-e, todos los cuales funcionan de manera similar. Con el avance de la tecnología, las nuevas generaciones de Cig-e no sólo han variado en su estética, sino que también permiten al usuario acomodar el dispositivo al utilizar diferentes líquidos y personalizar el elemento que se calienta ${ }^{22}$. Esto tiene, en último término, un impacto significativo en el usuario ya que de ello dependen las características de las partículas resultantes del proceso de vaporización del líquido, produciéndose en distintas cantidades y diversos tamaños, lo que les permite diferentes distribuciones en la vía aérea. También es responsable de la variabilidad de los niveles de los químicos y de nicotina que son entregados al usuario ${ }^{8,23-26}$.

Debido a la gran cantidad de distintos tipos de Cig-e y a la inmensa variedad de posibles combinaciones de los líquidos y saborizantes, es muy difícil determinar los potenciales efectos de la inhalación del aerosol generado, ya que no será muy exacto extrapolar los resultados de estudios que han utilizado Cig-e de distinta generación ${ }^{1}$.

\section{b) Contenido del líquido y del aerosol generado}

La composición del aerosol generado depende de los componentes del líquido que se "vaporiza", de las características eléctricas del elemento calentador, la temperatura alcanzada y las características de la mecha ${ }^{1}$. Se ha estimado que existen más de 7.000 diferentes líquidos, con distintos sabores y contenidos de nicotina ${ }^{27}$, casi todos ellos sin ningún tipo de regulación ${ }^{8}$.

\section{- Líquidos y bioproductos generados}

La gran mayoría de los líquidos de los Cig-e contienen glicerina vegetal, propilenglicol, saborizantes y otros aditivos, entre ellos, la nicotina ${ }^{28}$. La glicerina vegetal y el propilenglicol, junto con muchos de los saborizantes contenidos en los líquidos de los Cig-e, están permitidos para su consumo oral, pero no existen datos sobre su seguridad al ser inhalados por largo tiempo por seres humanos.

Los Cig-e generan un aerosol consistente en partículas finas y ultrafinas en fase gaseosa, las que en número y tamaño son similares a las producidas por los cigarrillos convencionales ${ }^{29-33}$. Un estudio $^{34}$ que cuantificó los potenciales componentes tóxicos del vapor de tres líquidos de Cig-e diferentes encontró seis constituyentes principales en los líquidos: propilenglicol, glicerina, nicotina, etanol, acetol y óxido de propileno. En los aerosoles generados por estos vaporizadores identificó 31 componentes, incluyendo nicotina, nicotireno, 
folmaldehido, acetaldehido, acreolina, acetol, diacetil y glicidol. Otros estudios han identificado especies oxígeno reactivas ${ }^{35}$, compuestos orgánicos volátiles, muchos de los cuales no aparecen en las listas de ingredientes ${ }^{36-38}$ y componentes tóxicos asociados a los saborizantes ${ }^{39}$. Además, se han reportado pequeñas cantidades de metales pesados $\mathrm{y}$, al menos, 20 carcinógenos conocidos (como el óxido de propileno, formaldehído ${ }^{40}$, glicidol $^{34}$ ) y agentes teratogénicos tanto en el líquido como en el vapor de los Cig-e $\mathrm{e}^{41-47}$.

Muchos de estos componentes identificados son irritantes de la vía aérea, como el propilengli$\operatorname{col}^{48}$, la acreolina ${ }^{49}$ y aldehídos ${ }^{29}$. La inhalación a largo plazo de algunos de estos componentes se ha asociado con disminución de la función pulmonar en seres humanos ${ }^{50} \mathrm{y}$ bronquiolitis obliterante, como es el caso del diacetil y/o acetilpropinil ${ }^{51,52}$. Por último, se ha identificado también en el aerosol del Cig-e, etilenglicol, el que no ha sido aprobado para su consumo en humanos ${ }^{53}$.

Si bien todos estos datos sugieren que el líquido y el vapor generado por los Cig-e contienen sustancias y partículas que pueden ser dañinas para la salud, hay que tener en cuenta que estos componentes se encuentran en concentraciones más bajas que las conocidas para causar toxicidad $^{1,54,55}$. Además, la formación de los aldehídos $\mathrm{y}$ de otros productos de descomposición térmica en el vapor del Cig-e se produce principalmente a altas temperaturas de vaporización ${ }^{56}$, lo que rara vez ocurre en la vida real, ya que la presencia de estos productos crea un sabor muy ácido al inhalar, lo que es evitado por los usuarios ${ }^{57}$.

\section{- Nicotina}

En los líquidos que contienen nicotina, la cuantía de su concentración sanguínea depende de varios factores, como la cantidad de nicotina contenida en el líquido, la temperatura alcanzada en la vaporización, el volumen del puff inhalado $^{26}$, la experiencia del usuario (aquellos experimentados pueden lograr niveles de nicotina similares a los alcanzados con los cigarrillos convencionales ${ }^{58}$ ) y la tecnología del dispositivo (en general, la entrega de nicotina es mayor en los dispositivos de última generación ${ }^{59}$ ).

Pero, ¿cuál es el riesgo de la exposición a largo plazo de la nicotina sobre la salud respiratoria? No existen muchos estudios realizados en seres humanos que puedan responder directamente la pregunta. Si bien algunas revisiones de estudios en animales y seres humanos han mostrado que la exposición a la nicotina afecta negativamente los sistemas cardiovascular, respiratorio, renal y reproductivo, como también puede tener efectos carcinogénicos al promover la tumorigénesis al afectar la proliferación celular, angiogénesis y apoptosis $^{60}$, la presencia de factores confundentes impiden llegar a conclusiones firmes. Por eso es que se ha propuesto que la nicotina por sí sola, más allá de sus efectos adictivos ${ }^{61}$, no contribuye directamente a las enfermedades relacionadas con el tabaquismo ${ }^{28}$. Esto último se puede deducir al examinar los efectos de la exposición a la nicotina en los estudios con las terapias de reemplazo nicotínico (TRN), las que no han mostrado mayores efectos adversos ${ }^{62,63}$. Por ejemplo, un estudio que evaluó los eventos adversos a las 52 semanas del uso de parches de nicotina, reportó que sólo el 4,7\% de los sujetos presentó algún evento adverso serio ${ }^{64}$, aunque hay que notar que en estos casos la administración de nicotina se hace en dosis relativamente bajas y por tiempo relativamente corto.

Existe amplia evidencia derivada de estudios en animales que la exposición a la nicotina tiene fuertes efectos negativos sobre el desarrollo pulmonar $\mathrm{fetal}^{8}$. Se ha comunicado que en los embriones expuestos, la nicotina estimula la arborización pulmonar y el crecimiento disináptico del pulmón en forma dosis-dependiente ${ }^{65,66}$, aumenta el tejido colágeno y conectivo en el epitelio de la vía aérea ${ }^{67,68}$, aumenta el volumen alveolar con disminución en la superficie alveolar ${ }^{69,70}$, disminuye los flujos espiratorios forzados ${ }^{71-73} \mathrm{y}$ aumenta la reactividad de la vía aérea en los recién nacidos ${ }^{71,74,75}$, entre otros efectos deletéreos. Pero no está claro si todos estos hallazgos pueden traspasarse a los seres humanos. Por ejemplo, un estudio realizado en 402 embarazadas fumadoras, las que fueron aleatorizadas a usar parches de nicotina versus placebo durante el embarazo, mostró que la exposición a la nicotina no alteró el peso de nacimiento ni la frecuencia de eventos adversos serios de la madre, del parto ni del recién nacido. Sólo se encontró un aumento de la presión arterial diastólica $(8 \mathrm{mmHg})$ en las madres ${ }^{76}$.

\section{c) Comportamiento y experiencia del usuario}

Se ha visto que con mayor tiempo de uso, mayores concentraciones de nicotina se alcanzan en la sangre y, probablemente, también se inhalan mayores cantidades de los otros componentes ${ }^{77}$. Por tanto, es importante tener claro la experiencia del usuario al interpretar los resultados de los estudios, lo que generalmente no se evalúa.

\section{d) Traslado de los efectos encontrados en estudios "in vitro" y en animales al hombre}

Este es un aspecto sumamente importante a la hora de sopesar las evidencias. Prácticamente 
casi todo lo que sabemos hoy sobre los efectos del Cig-e proviene de estudios en animales o in vitro. En ellos, se ha establecido una relación potencial entre la exposición al vapor del Cig-e y los efectos pulmonares negativos. Se ha demostrado en estos tipos de estudios que la exposición a corto plazo al vapor del Cig-e puede inducir inflamación pulmonar y estrés oxidativo sistémico y pulmonar, acompañado de alteraciones en la función de la barrera epitelial pulmonar ${ }^{35,78}$. La exposición a largo plazo ha mostrado producir cambios característicos de la Enfermedad Pulmonar Obstructiva Crónica: EPOC (patología de la vía aérea, inflamación y destrucción enfisematosa pulmonar $)^{79} \mathrm{y}$ daño en el ADN con disminución en la sobrevida de las células ${ }^{80}$. Pero, el extrapolar los hallazgos de estos estudios al hombre no es simple. Por una parte, hay que tener en cuenta las diferencias interespecies, la comparabilidad de las dosis, los tiempos de exposición a los Cige y el modo de uso ${ }^{1}$. De hecho, en los estudios en animales e in vitro, la exposición al vapor generado por los Cig-e es diferente a la que están expuestas las células del usuario del Cig-e. Debido a esta gran heterogeneidad, también se hace difícil llegar a conclusiones firmes si nos basamos en este tipo de estudios ${ }^{1}$.

La evidencia real debe provenir de estudios en seres humanos, los que son escasos. La mayoría de ellos se limitan a los efectos de la exposición a corto plazo, evidenciándose una disminución en el óxido nítrico exhalado y aumento en la resistencia de la vía aérea ${ }^{81,82}$, consistente con algún efecto irritativo, pero no se ha demostrado ningún efecto significativo sobre la función pulmonar ${ }^{83}$. Una forma de aproximarse a los posibles efectos del uso a largo plazo de los Cig-e en seres humanos es el análisis de casos publicados. Una reciente revisión encontró 27 casos publicados. En 25 casos, el uso del Cig-e produjo efectos negativos y en 2, positivos. Entre los casos negativos, 6 fueron respiratorios (dos neumonías lipoídeas, una bronquiolitis, una neumonía eosinofilica agu$\mathrm{da}$, una neumonía con derrame pleural bilateral y una probable neumonitis por hipersensibilidad) y 2 cardiovasculares (fibrilación auricular paroxística e infarto agudo del miocardio). De los dos casos con efectos positivos, uno se dio en un paciente fumador que tenía una neutrofilia idiopática y que logró dejar de fumar con el Cig-e y desapareció la neutrofilia. El otro caso fue un paciente con colitis ulcerosa que presentó una remisión con el uso de Cig-e y también dejó de fumar. Hubo 3 muertes asociadas al uso de Cig-e, todas relacionadas a conductas suicidas (ingesta o uso intravenoso del líquido del Cig-e $)^{84}$.
En suma, no existe evidencia concluyente que permita afirmar que el uso del Cig-e sea dañino para la salud respiratoria tanto a corto como a largo plazo y, aunque no es posible cuantificar los riesgos a largo plazo del uso de los Cig-e, los datos disponibles sugieren que es poco probable que estos excedan al 5\% de aquellos asociados a los cigarrillos convencionales ${ }^{15}$. Pero esta afirmación se debe principalmente a que no existen estudios de diseño y calidad adecuados para esclarecer esta interrogante. Debemos recordar que el no encontrar evidencias de que algo es dañino, no nos debe conducir necesariamente a concluir que es seguro. Se necesitan más estudios longitudinales, aleatorizados y controlados para evaluar el impacto de los Cig-e sobre la salud respiratoria a largo plazo $^{85}$.

\section{Escenarios}

Al no existir evidencias concluyentes sobre los efectos del uso de Cig-e a largo plazo sobre la salud respiratoria en humanos, ¿cuál debería ser nuestra posición como médicos clínicos?

Una forma práctica de enfocar el problema es analizar los diversos escenarios en los que los pacientes pueden encontrarse al momento de requerir recomendaciones.

\section{1) Sujeto con o sin enfermedad pulmonar crónica, no fumador, que quiera utilizar el Cig-e}

Para evaluar bien este escenario, se debe considerar el uso de los Cig-e no comparándolo con los cigarrillos convencionales, sino que con el no uso de ningún producto nicotínico ${ }^{8}$. En este contexto, si bien el vapor de los Cig-e contiene mucha menor cantidad de nicotina, toxinas y carcinógenos que los cigarrillos convencionales, por lo que los riesgos atribuibles a la inhalación de ellos son probablemente bajos ${ }^{15}$, los efectos de la exposición a estos niveles bajos a largo plazo no se conocen ${ }^{86}$. En este sentido, cuando no hay certeza sobre los riesgos, aunque sean mínimos, se debería aplicar el principio de precaución ${ }^{21}$, es decir, no se debería recomendar su uso.

El otro aspecto que se debe tener en cuenta en este escenario y que refuerza la recomendación anterior es el poder adictivo de la nicotina. El consumo de nicotina en personas que no fuman cigarrillos convencionales tiene claramente el riesgo de promover la adicción por la nicotina ${ }^{1}$.

Debido a que los sujetos no fumadores que inician el uso de Cig-e son preferentemente ado- 
lescentes y adultos jóvenes ${ }^{6,87}$, los que son especialmente susceptibles a la adicción a la nicotina después del uso Cig-e debido a que sus cerebros están aún en desarrollo y particularmente sensibles a la nicotina ${ }^{88}$, el uso del Cig-e en ellos está fuertemente asociado al uso de otros productos que contienen tabaco, particularmente, cigarrillos convencionales ${ }^{7}$. De hecho, se ha visto que los Cig-e pueden aumentar las tasas de iniciación de cigarrillos convencionales en jóvenes que, por alguna razón, nunca habrían probado los cigarrillos convencionales ${ }^{11,89,90}$. Una revisión sistemática y meta-análisis demostró una fuerte evidencia de asociación entre el uso inicial de Cig-e y la subsecuente iniciación de fumar cigarrillos convencionales en adolescentes y adultos jóvenes de 14 a 30 años de edad ${ }^{132}$.

Por tanto, en este escenario nunca será recomendable el uso del Cig-e ${ }^{1}$.

\section{2) Sujeto fumador, sin enfermedad pulmonar crónica, que quiera cambiar el cigarrillo convencional por el Cig-e, o que quiera ocupar el Cig-e para fumar menos el cigarrillo convencional}

En este escenario, la probable principal razón para iniciar el uso de Cig-e podría ser la percepción del menor daño que potencialmente producen estos dispositivos al compararse con los cigarrillos convencionales ${ }^{1}$ debido, nuevamente, a la disminución a la exposición de los miles de químicos tóxicos y carcinogénicos derivados de la combustión del tabaco ${ }^{28}$. En cuanto a la nicotina, no existen evidencias que sugieran que la nicotina inhalada de los Cig-e tenga algún perfil de riesgo apreciablemente diferente a la nicotina entregada por otras rutas de absorción ${ }^{15}$. Por tanto, si bien teóricamente este escenario podría ser favorable para la recomendación del uso de Cig-e, existen dos problemas. El primero es que no hay estudios epidemiológicos ni clínicos que avalen esta conducta ${ }^{1}$, por lo que no hay evidencia de ningún tipo ni a favor ni en contra. Escasos estudios podrían ayudarnos, pero en ninguno de ellos los pacientes se ajustan a nuestro escenario. Por ejemplo, un estudio clínico prospectivo, aleatorizado y controlado ${ }^{91}$ a 1 año, que comparó el Cig-e de primera generación versus placebo en sujetos fumadores que no tenían intención de dejar de fumar, mostró una disminución significativa del uso de cigarrillos convencionales, tanto en los grupos que utilizaron el Cig-e como en el placebo (este grupo utilizó Cig-e sin nicotina). Pero estos pacientes no se ajustan a los de nuestro escenario porque no tenían intenciones de cambiar al Cig-e. Otro estudio, de diseño poblacional en jóvenes, mostró que la introducción de los Cig-e no se asoció con cambios en la declinación del consumo de los cigarrillos convencionales ${ }^{90}$, aunque tampoco estos sujetos estudiados se ajustan a nuestro escenario.

El segundo problema es que hay cierta evidencia que sugiere que el uso dual de Cig-e y cigarrillos convencionales podría estar asociado con mayor dependencia a la nicotina ${ }^{92}$ y sin disminución significativa en la exposición a carcinógenos y toxinas comparado con el uso de sólo cigarrillos convencionales ${ }^{93}$.

En suma, si bien el cambio de cigarrillos convencionales por Cig-e probablemente disminuye el riesgo de efectos deletéreos sobre la salud respiratoria, tampoco se debería recomendar su uso en este escenario debido a que no se conocen los efectos a largo plazo y a la existencia de cierta evidencia de efectos perjudiciales con el uso dual de cigarrillos convencionales más Cig-e.

\section{3) Sujeto fumador, sin enfermedad pulmonar crónica, que quiera ocupar el Cig-e para dejar de fumar el cigarrillo convencional}

La racionalidad de este escenario radica en que los Cig-e que contienen nicotina pueden disminuir el síndrome de abstinencia e imitar, por ser utilizados de manera similar al cigarrillo tradicional, muchos de los aspectos conductuales característicos de los fumadores ${ }^{94}$. Para evaluar bien este escenario, la eficacia del Cig-e como alternativa terapéutica para dejar de fumar debería compararse con los tratamientos aprobados para la cesación tabáquica ${ }^{1}$. Existe hasta hoy sólo un estudio clínico prospectivo, aleatorizado y controlado por placebo que compara directamente los Cig-e con los parches de nicotina ${ }^{95}$. El estudio, que incluyó 657 fumadores de 10 o más cigarrillos al día y que querían dejar de fumar, aleatorizó a tres grupos de tratamiento durante 12 semanas: Cig-e con $16 \mathrm{mg} / \mathrm{mL}$ de nicotina $a d$ libitum; Cig-e sin nicotina (placebo); parches de nicotina, $21 \mathrm{mg}$ al día. Los resultados no mostraron diferencias entre los grupos en cuanto a la proporción de pacientes que se mantuvieron en abstinencia continua durante 6 meses. Pero este estudio tiene dos limitaciones importantes que podrían disminuir la fortaleza de sus conclusiones. Primero, que el estudio no alcanzó poder estadístico suficiente para concluir superioridad de los Cig-e sobre los parches ni sobre el placebo. Segundo, a los individuos asignados aleatoriamente a los Cig-e se les proveyó de ellos, mientras que a los asignados aleatoriamente a los parches de nicotina, se les dio un vale para que los obtuviesen ellos mismos de la farmacia. Esta 
diferencia podría haber sesgado potencialmente el estudio contra los parches.

Se han publicado varios estudios de cohorte que han evaluado el papel de los Cig-e como herramienta para dejar de fumar, pero estos estudios tienen una serie de limitaciones. Primero, las limitaciones propias de este tipo de diseño de estudio. Segundo, al comparar dichos estudios, se ve que se incluyen distintos tipos de poblaciones y con metodologías muy variadas, además que la gran mayoría basa sus resultados de abstinencia en el autoreporte. De ahí que los resultados sean contradictorios entre ellos.

Con el fin de tratar de identificar conclusiones fuertes, basadas en la evidencia, sobre el papel real de los Cig-e como terapia útil para la cesación tabáquica, se han realizado revisiones sistemáticas y varios metaanálisis de todos estos estudios publicados.

Una revisión sistemática reciente, que evaluó 62 estudios relevantes, mostró que la mayoría de ellos encontró que los Cig-e, especialmente los de segunda generación, pueden aliviar los síntomas de deprivación, aunque la calidad de los estudios incluidos fue considerada baja, por lo que no se pueden extraer conclusiones certeras ${ }^{96}$.

El primer metaanálisis Cochrane ${ }^{97}$ incluyó 13 estudios, de los cuales sólo dos eran aleatorizados y controlados, el que acabamos de revisar ${ }^{95}$ y otro que ya mencionamos anteriormente ${ }^{91}$, aunque este último fue comparado con placebo. Los autores concluyeron que el uso de Cig-e con nicotina ayuda a dejar de fumar a los 6 meses, pero enfatizan la principal limitación del metaanálisis: que incluyó sólo dos estudios aleatorizados y controlados y escasos participantes. Esto llevó a los autores a concluir que la evidencia era de baja o muy baja calidad. Una actualización de este metaanálisis, que incluyó estudios publicados hasta enero de 2016, analizó los resultados con 11 nuevos estudios, pero ninguno de ellos fue aleatorizado y controlado, por lo que las conclusiones no cambiaron ${ }^{98}$.

Un segundo metaanálisis ${ }^{10}$ incluyó cinco estudios poblacionales (4 longitudinales y uno transversal) y concluye que el uso de Cig-e se asoció con una menor probabilidad de cesación, aunque la principal limitación del estudio, aparte de incluir estudios poblacionales, fue que no se ajustaron los resultados por grado de dependencia a la nicotina.

Un tercer metaanálisis ${ }^{99}$ incluyó seis estudios (los mismos dos estudios controlados más dos de cohorte y dos tranversales), encontrando que la proporción de individuos que dejaron de fumar con los Cig-e llegó al 20\%. Las principales limi- taciones de este metaanálisis fueron que los estudios incluidos resultaron ser muy heterogéneos debido a los diferentes diseños y a la variación de los sexos. Los autores comentan que sólo se pudo analizar la eficacia de los Cig-e con nicotina versus los sin nicotina y que no se pudo evaluar la eficacia de los Cig-e versus otras intervenciones para dejar de fumar debido a la falta de grupos comparadores en los estudios incluidos.

Un cuarto metaanálisis ${ }^{100}$, que incluyó 18 estudios observacionales de vida real, un estudio clínico aleatorizado y otro no aleatorizado, encontró un $28 \%$ de menor probabilidad de dejar de fumar en aquellos que utilizaron Cig-e versus los que no lo usaron. Las limitaciones de este metaanálisis son principalmente que incluyeron pacientes con $\mathrm{y}$ sin deseos de dejar de fumar y estudios con autoreporte de abstinencia ${ }^{101}$.

En suma, los metaanálisis no han arrojado resultados concluyentes para nuestro escenario debido a las serias limitaciones de cada uno de ellos, lo que debilita la fortaleza de sus conclusiones.

Si bien es por esta falta de evidencias que la USPSTF (U.S. Preventive Services Task Force) no recomienda el uso de Cig-e para la cesación tabáquica ${ }^{102}$, esto podría no ser cierto para todos los fumadores. La motivación correcta es la principal causa que hace que el fumador deje de fumar y la forma más común con la que los fumadores lo logran es la decisión espontánea, generalmente provocada por algún gatillante particular ${ }^{103}$. Por tanto, podría ser probable que la oportunidad de dejar definitivamente el cigarrillo convencional con el uso del Cig-e fuese mayor si el fumador eligiera esto conscientemente ${ }^{104}$. Se podría, entonces, afirmar que, en sujetos fumadores que no han podido dejar de fumar con las terapias aprobadas existentes o que no las toleran $\mathrm{y}$ que quieren dejar de fumar cigarrillos convencionales con el uso de Cig-e a corto plazo, podría ser una alternativa razonable para dejar de fu$\operatorname{mar}^{62} \mathrm{y}$, como la terapia combinada en la cesación tabáquica es más efectiva que la monoterapia ${ }^{105}$, no es inverosímil la posibilidad de utilizar los Cig-e junto a la vareniclina, como parte de una terapia combinada. Aunque esta proposición no tiene evidencia alguna.

Es importante recalcar que esta proposición requiere que el uso de los Cig-e sea considerado como una terapia, lo que requeriría, al menos, tres condiciones: primero, que sea utilizado a corto plazo; segundo que sea utilizado en forma diaria, ya que se ha visto que aquellos que utilizan los Cig-e diariamente tienen mayor probabilidad de dejar de fumar cigarrillos convencionales que 
aquellos que no los utilizan diariamente ${ }^{106,107}$; tercero, que no sea de venta libre ( $\sin$ receta), ya que esto podría tener los mismos efectos que se han visto con las terapias de reemplazo nicotínico, las que cuando se utilizan bajo prescripción médica tienen mayores tasas de cesación tabáquica que cuando se utilizan libremente ${ }^{108}$.

\section{4) Sujeto fumador, con enfermedad pulmonar crónica, que quiera ocupar el Cig-e para dejar de fumar el cigarrillo convencional}

Este escenario tiene las mismas consideraciones descritas en el escenario anterior, pero, debido a los conocidos efectos deletéreos del tabaquismo activo en pacientes con enfermedades respiratorias crónicas, en estas poblaciones se hace más necesario el lograr la abstinencia. A pesar de la importancia del cese tabáquico en las enfermedades respiratorias crónicas ${ }^{109,110}$ y a lo particularmente difícil de hacerlo en los pacientes con asma, EPOC y cáncer pulmonar ${ }^{111,112} \mathrm{y}$, aunque algunos intentos se han hecho ${ }^{112}$, llama la atención que no existan estrategias de tratamiento antitabáquicas dirigidas específicamente a estas poblaciones $^{113}$.

Como toda terapia antitabáquica exitosa, y con mayor razón en este escenario, estas deben incluir consultas de seguimiento frecuentes y la combinación de consejería médica intensiva con apoyo conductual individual y tratamiento farmacológico agresivo ${ }^{114,115}$. La terapia farmacológica óptima debe comprender la combinación de agentes farmacológicos, como alguna terapia de reemplazo nicotínico (TRN) más vareniclina o bupropión, por al menos 3 meses. Si no se logra la abstinencia o si el paciente recae, debería intentarse un retratamiento ${ }^{116}$. Y en este retratamiento es donde podrían incluirse a los Cig-e. $\mathrm{Si}$ bien muchos autores sugieren que los Cig-e pueden servir de ayuda en pacientes con enfermedades respiratorias crónicas como parte de la terapia de cesación tabáquica ${ }^{111,117,118}$, la eficacia y seguridad al usar los Cig-e, ya sea como TRN única o asociada a otra TRN, más varineclina o bupoprion no se conoce ${ }^{119}$. No existen estudios aleatorizados y controlados que hayan explorado este escenario y algunos estudios observacionales han arrojado dudas sobre la utilidad de los Cig-e para facilitar la cesación tabáquica en pacientes con cáncer ${ }^{92}$.

Por tanto, la recomendación podría ser, al igual que en el escenario anterior, utilizar el Cig-e sólo después que el paciente haya intentado dejar de fumar con las terapias convencionales sin resultados positivos y con las mismas consideraciones que el resto de las TRN.

\section{5) Sujeto fumador, con enfermedad pulmonar crónica, que quiera cambiar el cigarrillo convencional por el Cig-e, o que quiera ocupar el Cig-e para fumar menos el cigarrillo convencional}

La razón de este escenario es por el daño demostrado que producen las sustancias del humo del cigarrillo convencional en las enfermedades respiratorias crónicas ${ }^{112}$, por lo que para evaluar este escenario se debe comparar la exposición a toxinas y carcinógenos entre el cigarrillo convencional y el Cig-e. Se ha visto que, luego de cambiar de cigarrillos convencionales al Cig-e, la exposición a nicotina sigue siendo la misma, pero la exposición a carcinógenos y tóxicos se disminuye sustancialmente ${ }^{120}$. Pero, como ya mencionamos, esto sucede si los usuarios utilizan sólo Cig-e y no cuando los asocian con cigarrillos convencionales ${ }^{93}$. Por tanto, nuestro escenario se reduce sólo a aquellos que quieran cambiar completamente el cigarrillo convencional por el Cig-e.

Existen escasos estudios que hayan evaluado este escenario y ninguno, hasta la fecha, controlado y aleatorizado. Un pequeño estudio no controlado en 18 asmáticos fumadores mostró que después de dos años de haber cambiado a Cig-e mejoró la función pulmonar y los síntomas con una disminución significativa en el uso de cigarrillos convencionales ${ }^{121}$. Un estudio retrospectivo ${ }^{122}$ de pacientes con EPOC mostró que aquellos que utilizaron Cig-e lograron disminuir en forma significativa el consumo de cigarrillos convencionales e incluso dejarlos por completo a los dos años de seguimiento. Se observó también que los que utilizaron Cig-e disminuyeron significativamente los episodios de exacerbaciones. Existe un estudio en curso para evaluar la efectividad de los Cig-e como herramienta para disminuir los riesgos relacionados con el consumo de cigarrillos convencionales sobre el cáncer pulmonar y poder así incorporar estos dispositivos como parte del programa de tamizaje de cáncer pulmonar ${ }^{123}$.

Por tanto, en este escenario se recomienda cambiar el cigarrillo convencional por el Cig-e.

\section{6) Embarazada que desea cambiar del cigarrillo convencional al Cig-e para evitar los riesgos sobre su hijo}

En este escenario se deben considerar los efectos de la nicotina sobre el embarazo y, si bien los datos sobre los efectos de la nicotina derivan primariamente de estudios en animales, las grandes similitudes entre los hallazgos en monos, ratas y ovejas, comparado con los hallazgos en mujeres embarazadas que fuman cigarrillos convenciona- 
les, apoyan un efecto directo de la nicotina sobre el desarrollo pulmonar fetal ${ }^{8}$.

No hay estudios aleatorizados y controlados en relación con el uso de los Cig-e en las mujeres embarazadas, por lo que no se conocen sus efectos sobre la salud materna o fetal ${ }^{124,125}$, por lo que sus efectos podrían teóricamente asimilarse a los efectos de la nicotina cuando se utiliza como TRN. Un metaanálisis de estudios randomizados y controlados con y sin tratamiento conductual en embarazadas concluyó que no hay evidencia suficiente para concluir la seguridad ni efectividad del uso de parches de nicotina durante el embarazo $^{126}$. Un gran estudio, no incluido en el metaanálisis, que randomizó a más de mil embarazadas a parches de nicotina $15 \mathrm{mg}$ por $16 \mathrm{~h}$ versus placebo, no encontró aumento en el riesgo de embarazo adverso ni de desenlaces del parto entre ambos grupos, aunque la principal limitación del estudio fue la baja adherencia al uso de los parches ${ }^{127}$.

Por tanto, debido a que no se conoce la cantidad de nicotina segura para su uso durante el embarazo y a la ausencia de demostración de la eficacia y seguridad a largo plazo del uso de los Cig-e durante el embarazo, no se recomien$\mathrm{da}^{124,128}$.

\section{Resumen y conclusiones}

Los Cig-e son mucho más seguros que los cigarrillos convencionales ya que entregan mucha menor cantidad de toxinas y carcinógenos, aunque el efecto a largo plazo sobre las vías aéreas y el pulmón de esta mínima exposición no se conoce. La cantidad de nicotina entregada por los Cige de últimas generaciones no son diferentes a las entregadas por los cigarrillos convencionales y, si bien no hay datos claros de efectos deletéreos sobre la salud respiratoria debido a la nicotina, esto podría depender de las dosis, del tiempo y el momento (vida prenatal o vida temprana) de exposición, sobre lo cual no hay mucha evidencia en seres humanos.

Existen actualmente varias áreas de incertidumbre y preocupación en cuanto al uso a largo plazo de los Cig-e. Primero, los efectos de la inhalación del vapor generado sobre la salud respiratoria. Segundo, la posibilidad cierta de que representen un puente hacia el consumo posterior de cigarrillos convencionales, especialmente en los jóvenes ${ }^{132}$. Tercero, la escasa regulación y control de calidad de los contenidos de los cada vez más numerosos líquidos y saborizantes disponibles ${ }^{8,94}$. Cuarto, los efectos potenciales de la exposición secundaria y terciaria ${ }^{34,86,129}$.
Entonces, ante la pregunta de si deberíamos recomendar el uso de los Cig-e, la respuesta es definitivamente no en la población general. Pero en ciertas situaciones, y siempre después de haber intentado las terapias de cesación tabáquica adecuadas y aprobadas, como en el caso del fumador que no ha podido dejar de fumar luego de varios intentos terapéuticos, o fumadores con enfermedad pulmonar crónica en esa misma situación, el uso del Cig-e, bajo los mismos principios que las TRN, podría considerarse ${ }^{94}$. En estos casos, más que prohibir los Cig-e, debería exigirse que se regularan como productos estandarizados y que se les exigiera también los mismos requerimientos de seguridad que a los otros tratamientos antitabaco $^{130}$. Bajo este contexto, su uso debería ser sólo bajo estricta supervisión de personal entrenado en terapia de cesación tabáquica, por lo que su venta debería ser estrictamente regulada ${ }^{60}$ y siempre evitarse lo máximo posible el uso dual de Cig-e más cigarrillos convencionales.

La evidencia actual sugiere que existen variantes genéticas que pueden predecir la falla en la cesación tabáquica y que la efectividad de la farmacoterapia de cesación es modulada por ciertos biomarcadores ${ }^{131}$. Quizás en un futuro, y con el enfoque de la medicina de precisión, se desarrollen biomarcadores útiles que puedan predecir la efectividad de los Cig-e como terapia de cesación tabáquica en la población general.

\section{Bibliografía}

1.- DINAKAR C, O'CONNOR G T. The Health Effects of Electronic Cigarettes. N Engl J Med 2016; 375: 137281.

2.- LEDUC C, QUOIX E. Is there a role for e-cigarettes in smoking cessation? Ther Adv Respir Dis 2016; 10: 130-13.

3.- ORELLANA-BARRIOS M A, PAYNE D, MULKEY Z, NUGENT K. Electronic Cigarettes-A Narrative Review for Clinicians. Am J Med 2015; 128: 674-81.

4.- "Vapea Chile", Página web: www.vapeachile.cl/ preguntas-frecuentes.

5.- ARRAZOLA R A, NEFF L J, KENNEDY S M, HOLDER-HAYES E, JONES C D. Tobacco Use Among Middle and High School Students-United States, 2013. MMWR Morb Mortal Wkly Rep 2014; 63: 1021-6.

6.- ARRAZOLA R A, SINGH T, COREY C G, HUSTEN C G, NEFF L J, APELBERG B J et al. Tobacco Use Among Middle and High School Students-United States, 2011-2014. MMWR Morb Mortal Wkly Rep 2015; 64: 381-5.

7.- U.S. DEPARTMENT OF HEALTH AND HUMAN SERVICES. E-Cigarette Use Among Youth and Young Adults. A Report of the Surgeon General. Atlanta, 
GA: U.S. Department of Health and Human Services, Centers for Disease Control and Prevention, National Center for Chronic Disease Prevention and Health Promotion, Office on Smoking and Health, 2016.

8.- SPINDEL E R, MCEVOY C T. The Role of Nicotine in the Effects of Maternal Smoking during Pregnancy on Lung Development and Childhood Respiratory Disease Implications for Dangers of E-Cigarettes. Am J Respir Crit Care Med 2016; 193: 486-94.

9.- GOSTIN L O, GLASNER A Y. E-cigarettes, vaping, and youth. JAMA 2014; 312: 595-6.

10.- GRANA R, BENOWITZ N, GLANTZ S. E-cigarettes: a scientific review. Circulation 2014; 129: 1972-86.

11.- LEVENTHAL A M, STRONG D R, KIRKPATRICK M G, UNGER J B, SUSSMAN S, RIGGS N R et al. Association of Electronic Cigarette Use With Initiation of Combustible Tobacco Product Smoking in Early Adolescence. JAMA 2015; 314: 700-7.

12.- PUBLIC HEALTH ENGLAND. E-cigarettes: an evidence update. A report commissioned by Public Health England. August 2015; PHE publications gateway number: 2015260.

13.- ETTER J F. E-cigarettes: methodological and ideological issues and research priorities. BMC Medicine 2015; 13: 32 .

14.- PUBLIC HEALTH ENGLAND. Underpinning evidence for the estimate that e-cigarette use is around $95 \%$ safer than smoking: authors' note. August 2015; PHE publications gateway number: 2015260.

15.- ROYAL COLLEGE OF PHYSICIANS. Nicotine without smoke: Tobacco harm reduction. London: RCP, 2016.

16.- ASH briefing on electronic cigarettes. Disponible en: http://ash.org.uk/stopping-smoking/ash-briefing-onelectronic-cigarettes-2/

17.- DIRECTIVE 2014/40/EU OF THE EUROPEAN PARLIAMENT AND OF THE COUNCIL.Disponible en: http://eur-lex.europa.eu/legal-content/EN/TXT/HTML /?uri=CELEX:32014L0040\&from=FR

18.- US FOOD \& DRUG ADMINISTRATION. Disponible en: http://www.fda.gov/NewsEvents/Newsroom/PressAnnouncements/ucm394667.htm

19.- WORLD HEALTH ORGANIZATION. Electronic nicotine delivery systems. 2014. http://apps.who.int/ $\mathrm{gb} / \mathrm{fctc} / \mathrm{PDF} / \mathrm{cop} 6 /$ FCTC_COP6_10Rev1-en.pdf?ua=1

20.- EUROPEAN COMMISSION. E-cigarettes myth buster. 2015.Disponible en: http://ec.europa.eu/health/ tobacco/docs/tobacco_mythbuster_en.pdf

21.- MCKEE M, CAPEWELL S. Evidence about electronic cigarettes: a foundation built on rock or sand? BMJ 2015; 351: h4863.

22.- FARSALINOS K E, POLOSA R. Safety evaluation and risk assessment of electronic cigarettes as tobacco cigarette substitutes: a systematic review. Ther Adv Drug Saf 2014; 5: 67-86.

23.- BROWN C J, CHENG J M. Electronic cigarettes: product characterisation and design considerations. Tobacco Control 2014; 23 (Suppl 2): ii4-10.

24.- BHATNAGAR A, WHITSEL L P, RIBISL K M, BULLEN C, CHALOUPKA F, PIANO M R et al. Electronic cigarettes: a policy statement from the American Heart Association. Circulation 2014; 130 (16): 141836.

25.- KOSMIDER L, SOBCZAK A, FIK M, KNYSAK J, ZACIERA M, KUREK J et al. Carbonyl compounds in electronic cigarette vapors: effects of nicotine solvent and battery output voltage. Nicotine \& Tobacco Research 2014; 16: 1319-26.

26.- TALIH S, BALHAS Z, EISSENBERG T, SALMAN R, KARAOGHLANIAN N, EL HELLANI A et al. Effects of user puff topography, device voltage, and liquid nicotine concentration on electronic cigarette nicotine yield: measurements and model predictions. Nicotine Tob Res 2015; 17: 150-7.

27.- ZHU S H, SUN J Y, BONNEVIE E, CUMMINS S E, GAMST A, YIN L et al. Four hundred and sixty brands of e-cigarettes and counting: implications for product regulation. Tobacco Control 2014; 23 (Suppl 3): iii3-9.

28.- HSIEH F H. Evidence $v s$ advocacy in the e-cigarette debate: to vape or not to vape, that is the question. Ann Allergy Asthma Immunol 2016; 116: 89-91.

29.- GEISS O, BIANCHI I, BARAHONA F, BARREROMORENO J. Characterisation of mainstream and passive vapours emittedby selected electronic cigarettes. Int J Hyg Environ Health 2015; 218: 169-80.

30.- FUOCO F C, BUONANNO G, STABILE L, VIGO P. Influential parameters on particle concentration and size distribution in the mainstream of e-cigarettes. Environ Pollut 2014; 184: 523-9.

31.- SCHOBER W, SZENDREI K, MATZEN W, OSIANDER-FUCHS H, HEITMANN D, SCHETTGEN T, et al. Use of electronic cigarettes (e-cigarettes) impairs indoor air quality and increases FeNO levels of ecigarette consumers. Int J Hyg Environ Health 2014; 217: 628-37.

32.- SCHRIPP D, MARKEWITZ D, UHDE E, SALTHAMMER T. Does e-cigarette consumption cause passive vaping? Indoor Air 2013; 23: 25-31.

33.- INGEBRETHSEN B J, COLE S K, ALDERMAN S L. Electronic cigarette aerosol particle size distribution measurements. Inhal Toxicol 2012; 24: 976-84.

34.- SLEIMAN M, LOGUE J M, MONTESINOS N, RUSSELL M L, LITTER M I, GUNDEL L A et al. Emissions from Electronic Cigarettes: Key Parameters Affecting the Release of Harmful Chemicals. Environ Sci Technol 2016; 50: 9644-51.

35.- LERNER C A, SUNDAR I K, YAO H, GERLOFF J, OSSIP D J, MCINTOSH S et al. Vapors produced by electronic cigarettes and e-juices with flavoring induce toxicity, oxidative stress, and inflammatory response in lung epithelial cells and in mouse lung. PLoS One 2015; 10: e0116732. 
36.- VARLET V, FARSALINOS K, AUGSBURGER M, THOMAS A, ETTER J F. Toxicity assessment of refill liquids for electronic cigarettes. Int J Environ Res Public Health 2015; 12: 4796-815.

37.- $\mathrm{OH} \mathrm{J} \mathrm{A,} \mathrm{SHIN} \mathrm{H} \mathrm{S.} \mathrm{Identification} \mathrm{and} \mathrm{quantification}$ of several contaminated compounds in replacement liquids of electronic cigarettes by gas chromatographymass spectrometry. J Chromatogr Sci 2015;53:841-8.

38.- LISKO J G, TRAN H, STANFILL S B, BLOUNT B C, WATSON C H. Chemical composition and evaluation of nicotine, tobacco alkaloids, $\mathrm{pH}$, and selected flavors in e-cigarette cartridges and refill solutions. Nicotine Tob Res 2015;17:1270-8.

39.- BEHAR R Z, DAVIS B, WANG Y, BAHL V, LIN S, TALBOT P. Identification of toxicants in cinnamonflavored electronic cigarette refill fluids. Toxicol In Vitro 2014; 28: 198-208

40.- DRUMMOND M B, UPSON D. Electronic cigarettes: potential harms and benefits. Ann Am Thorac Soc 2014; 11: 236-42.

41.- GRANA R A, LING P M. Smoking revolution: a content analysis of electronic cigarette retail websites. Am J Prev Med 2014; 46: 395-403.

42.- GONIEWICZ M L, KNYSAK J, GAWRON M, KOSMIDER L, SOBCZAK A, KUREK J et al. Levels of selected carcinogens and toxicants in vapour from electronic cigarettes. Tob Control 2014; 23: 133-9.

43.- FARSALINOS K E, VOUDRIS V, POULAS K. Are metals emitted from electronic cigarettes a reason for health concern? A risk assessment analysis of currently available literature. Int J Environ Res Public Health 2015; 12: 5215-32.

44.- WILLIAMS M, VILLARREAL A, BOZHILOV $\mathrm{K}$, LIN S, TALBOT P. Metal and silicate particles including nanoparticles are present in electronic cigarette cartomizer fluid and aerosol. PLoS One 2013; 8: e57987.

45.- LERNER C A, SUNDAR I K, WATSON R M, ELDER A, JONES R, DONE D et al. Environmental health hazards of e-cigarettes and their components: oxidants and copper in e-cigarette aerosols. Environ Pollut 2015; 198: 100-7.

46.- CHENG T. Chemical evaluation of electronic cigarettes. Tob Control 2014; 23 (Suppl. 2): ii11-7.

47.- BAHL V, LIN S, XU N, DAVIS B, WANG Y H, TALBOT P. Comparison of electronic cigarette refill fluid cytotoxicity using embryonic and adult models. Reprod Toxicol 2012; 34: 529-37.

48.- WIESLANDER G, NORBACK D, LINDGREN T. Experimental exposure to propylene glycol mist in aviation emergency training: acute ocular and respiratory effects. Occup Environ Med 2001; 58: 649-55.

49.- SRIVASTAVA S, SITHU S D, VLADYKOVSKAYA E, HABERZETTL P, HOETKER D J, SIDDIQUI M A et al. Oral exposure to acrolein exacerbates atheroscle-rosis in apoE-null mice. Atherosclerosis 2011; 215: 301-8.
50.- VARUGHESE S, TESCHKE K, BRAUER M, CHOW Y, VAN NETTEN C, KENNEDY S M. Effects of theatrical smokes and fogs on respiratory health in the entertainment industry. Am J Ind Med 2005; 47: 411-8.

51.- FARSALINOS K E, KISTLER K A, GILLMAN G, VOUDRIS V. Evaluation of electronic cigarette liquids and aerosol for the presence of selected inhalation toxins. Nicotine Tob Res 2015; 17: 168-74.

52.- KAISARA M A, PRASADA S, LILESA T, CUCULLO L. A decade of e-cigarettes: Limited research \& unresolved safety concerns. Toxicology 2016; 365: 67-75.

53.- HUTZLER C, PASCHKE M, KRUSCHINSKI S, HENKLER F, HAHN J, LUCH A. Chemical hazards present in liquids and vapors of electronic cigarettes. Arch Toxicol 2014; 88: 1295-308.

54.- MCAULEY T R, HOPKE P K, ZHAO J, BABAIAN $\mathrm{S}$. Comparison of the effects of e-cigarette vapor and cigarette smoke on indoor air quality. Inhal Toxicol 2012; $24: 850-7$

55.- GHOSH S, DRUMMOND M B. Electronic cigarettes as smoking cessation tool: are we there? Curr Opin Pulm Med 2017; 23: 111-6.

56.- FARSALINOS K E, VOUDRIS V, POULAS K. Ecigarettes generate high levels of aldehydes only in 'dry puff' conditions. Addiction 2015; 110: 1352-6.

57.- HAJEK P. Commentary on Farsalinos et al: Ecigarettes do not expose users to dangerous levels of aldehydes. Addiction 2015; 110: 1357-8.

58.- FARSALINOS K E, SPYROU A, STEFOPOULOS C, TSIMOPOULOU K, KOURKOVELI P, TSIAPRAS D et al. Nicotine absorption from electronic cigarette use: comparison between experienced consumers (vapers) and na ive users (smokers). Sci Rep 2015; 5: 11269.

59.- FARSALINOS K E, SPYROU A, TSIMOPOULOU $\mathrm{K}$, STEFOPOULOS C, ROMAGNA G, VOUDRIS $\mathrm{V}$. Nicotine absorption from electronic cigarette use: comparison between first and new-generation devices. Sci Rep 2014; 4: 4133.

60.- MISHRA A, CHATURVEDI P, DATTA S, SINUKUMAR S, JOSHI P, GARG A. Harmful effects of nicotine. Indian J Med Paediatr Oncol 2015; 36: 24-31.

61.- BENOWITZ N L. Clinical pharmacology of nicotine: implications for understanding, preventing, and treating tobacco addiction. Clin Pharmacol Ther 2008; 83: 53141.

62.- BALDASSARRI S R, TOLL B A, LEONE F T. A Comprehensive Approach to Tobacco Dependence Interventions. J Allergy Clin Immunol Pract 2015; 3: 481-8.

63.- HUBBARD R, LEWIS S, SMITH C, GODFREY C, SMEETH L, FARRINGTON $P$ et al. Use of nicotine replacement therapy and the risk of acute myocardial infarction, stroke, and death. Tob Control 2005; 14: 416-21.

64.- SCHNOLL R A, GOELZ P M, VELUZ-WILKINS A, BLAZEKOVIC S, POWERS L, LEONE F T et al. 
Long-term Nicotine Replacement Therapy. A Randomized Clinical Trial. JAMA Intern Med 2015; 175 : 504-11.

65.- WONGTRAKOOL C, ROSER-PAGE S, RIVERA H N, ROMÁN J. Nicotine alters lung branching morphogenesis through the alpha7 nicotinic acetylcholine receptor. Am J Physiol Lung Cell Mol Physiol 2007; 293: L611-8.

66.- WUENSCHELL C W, ZHAO J, TEFFT J D, WARBURTON D. Nicotine stimulates branching and expression of SP-A and SP-C mRNAs in embryonic mouse lung culture. Am J Physiol 1998; 274: L165-70.

67.- SEKHON H S, KELLER J A, PROSKOCIL B J, MARTIN E L, SPINDEL E R. Maternal nicotine exposure upregulates collagen gene expression in fetal monkey lung: association with alpha7 nicotinic acetylcholine receptors. Am J Respir Cell Mol Biol 2002; 26: 31-41.

68.- SEKHON H S, PROSKOCIL B J, CLARK J A, SPINDEL E R. Prenatal nicotine exposure increases connective tissue expression in foetal monkey pulmonary vessels. Eur Respir J 2004;23:906-15.

69.- SEKHON H S, JIA Y, RAAB R, KURYATOV A, PANKOW J F, WHITSETT J A, et al Prenatal nicotine increases pulmonary alpha7 nicotinic receptor expression and alters fetal lung development in monkeys. J Clin Invest 1999; 103: 637-47.

70.- MARITZ G S, WOOLWARD K M, DU TOIT G. Maternal nicotine exposure during pregnancy and development of emphysema-like damage in the offspring. S Afr Med J 1993; 83: 195-8.

71.- WONGTRAKOOL C, WANG N, HYDE D M, ROMAN J, SPINDEL E R. Prenatal nicotine exposure alters lung function and airway geometry through a7 nicotinic receptors. Am J Respir Cell Mol Biol 2012; 46: 695-702.

72.- PROSKOCIL B J, SEKHON H S, CLARK J A, LUPO S L, JIA Y, HULL W M et al. Vitamin C prevents the effects of prenatal nicotine on pulmonary function in newborn monkeys. Am J Respir Crit Care Med 2005; 171: 1032-9.

73.- SEKHON H S, KELLER J A, BENOWITZ N L, SPINDEL E R. Prenatal nicotine exposure alters pulmonary function in newborn rhesus monkeys. Am J Respir Crit Care Med 2001; 164: 989-94.

74.- SANDBERG K L, PINKERTON K E, POOLE S D, MINTON P A, SUNDELL H W. Fetal nicotine exposure increases airway responsiveness and alters airway wall composition in young lambs. Respir Physiol Neurobiol 2011; 176: 57-67.

75.- LIU J, SAKURAI R, O'ROARK E M, KENYON N J, TORDAY J S, REHAN V K. PPARg agonist rosiglitazone prevents perinatal nicotine exposure-induced asthma in rat offspring. Am J Physiol Lung Cell Mol Physiol 2011; 300: L710-7.

76.- BERLIN I, GRANGÉ G, JACOB N, TANGUY M L. Nicotine patches in pregnant smokers: randomised, placebo controlled, multicentre trial of efficacy. BMJ 2014; 348: g1622.

77.- HAJEK P, GONIEWICZ M L, PHILLIPS A, MYERS SMITH K, WEST O, MCROBBIE H. Nicotine intake from electronic cigarettes on initial use and after 4 weeks of regular use. Nicotine Tob Res 2015; 17: 1759.

78.- SCHWEITZER K S, CHEN S X, LAW S, VAN DEMARK M, POIRIER C, JUSTICE M J et al. Endothelial disruptive proinflammatory effects of nicotine and e-cigarette vapor exposures. Am J Physiol Lung Cell Mol Physiol 2015; 309: L175-87.

79.- GARCIA-ARCOS I, GERAGHTY P, BAUMLIN N, CAMPOS M, DABO A J, JUNDI B et al. Chronic electronic cigarette exposure in mice induces COPD in a nicotine-dependent manner. Thorax 2016; 71: 1119-29.

80.- YU V, RAHIMY M, KORRAPATI A, XUAN Y, ZOU A E, KRISHNAN A R et al. Electronic cigarettes induce DNA strand breaks and cell death independently of nicotine in cell lines. Oral Oncol 2016; 52: 58-65.

81.- VARDAVAS C I, ANAGNOSTOPOULOS N, KOUGIAS M, EVANGELOPOULOU V, CONNOLLY G N, BEHRAKIS P K et al. Short-term pulmonary effects of using an electronic cigarette: Impact on respiratory flow resistance, impedance, and exhaled nitric oxide. Chest 2012; 141: 1400-6.

82.- MARINI S, BUONANNO G, STABILE L, FICCO G. Short-term effects of electronic and tobacco cigarettes on exhaled nitric oxide. Toxicol Appl Pharmacol 2014; 278: 9-15.

83.- FLOURIS A D, CHORTI M S, POULIANITI K P, JAMURTAS A Z, KOSTIKAS K, TZATZARAKIS M $\mathrm{N}$ et al. Acute impact of active and passive electronic cigarette smoking on serum cotinine and lung function. Inhal Toxicol 2013; 25: 91-101.

84.- HUA M, TALBOT P. Potential health effects of electronic cigarettes: A systematic review of case reports. Prev Med Reports 2016; 4: 169-78.

85.- GLASSER A M, COLLINS L, PEARSON J L, ABUDAYYEH H, NIAURA R S, ABRAMS D B et al. Overview of Electronic Nicotine Delivery Systems: A Systematic Review. Am J Prev Med 2017; 52: e33-e66.

86.- MORRIS P B, FERENCE B A, JAHANGIR E, FELDMAN D N, RYAN J J, BAHRAMI H, et al. Cardiovascular Effects of Exposure to Cigarette Smoke and Electronic Cigarettes Clinical Perspectives From the Prevention of Cardiovascular Disease Section Leadership Council and Early Career Councils of the American College of Cardiology. J Am Coll Cardiol 2015; 66: 1378-91.

87.- MCMILLEN R C, GOTTLIEB M A, SHAEFER R $\mathrm{M}$, WINICKOFF J P, KLEIN J D. Trends in electronic cigarette use among U.S. adults: use is increasing in both smokers and nonsmokers. Nicotine Tob Res 2015; 17: 1195-220.

88.- US DEPARTMENT OF HEALTH AND HUMAN 
SERVICES. The health consequences of smoking-50 years of progress: a report of the Surgeon General. Disponible en: http://www.surgeongeneral.gov/library/ reports/50-years-of-progress/index.html.

89.- RIGOTTI N A. e-Cigarette Use and Subsequent Tobacco Use by Adolescents New Evidence About a Potential Risk of e-Cigarettes. JAMA 2015; 314: 673-4.

90.- DUTRA LM, GLANTZ S A. E-cigarettes and National Adolescent Cigarette Use: 2004-2014. Pediatrics 2017; 139: e20162450.

91.- CAPONNETTO P, CAMPAGNA D, CIBELLA F, MORJARIA J, CARUSO M, RUSSO C et al. EffiCiency and safety of an eLectronic cigarette (ECLAT) as tobacco cigarettes substitute: a prospective 12-month randomized control design study. PloS One 2013; 8: e66317.

92.- BORDERUD S P, LI Y, BURKHALTER J E, SHEFFER C E, OSTROFF J S. Electronic cigarette use among patients with cancer: characteristics of electronic cigarette users and their smoking cessation outcomes. Cancer 2014; 120: 3527-35.

93.- SHAHAB L, GONIEWICZ M J, BLOUNT B C, BROWN J, MCNEILL A, ALWIS K U et al. Nicotine, Carcinogen, and Toxin Exposure in Long-Term ECigarette and Nicotine Replacement Therapy Users. A Cross-sectional Study. Ann Intern Med 2017; 166: 390-400

94.- YEH J S, BULLEN C, GLANTZ S A. E-Cigarettes and Smoking Cessation. N Engl J Med 2016; 374: 2172-4.

95.- BULLEN C, HOWE C, LAUGESEN M, MCROBBIE H, PARAG V, WILLIMAN J et al. Electronic cigarettes for smoking cessation: a randomised controlled trial. Lancet 2013; 382: 1629-37.

96.- MALAS M, VAN DER TEMPEL J, SCHWARTZ R, MINICHIELLO A, LIGHTFOOT C, NOORMOHAMED A et al. Electronic Cigarettes for Smoking Cessation: A Systematic Review. Nicotine Tob Res 2016; 18: 1926-36

97.- MCROBBIE H, BULLEN C, HARTMANN-BOYCE J, HAJEK P. Electronic cigarettes for smoking cessation and reduction. Cochrane Database Syst Rev 2014; 12: CD010216.

98.- HARTMANN-BOYCE J , MCROBBIE H, BULLEN C, BEGH R, STEAD L F, HAJEK P. Electronic cigarettes for smoking cessation. Cochrane Database of Systematic Reviews 2016; 9: CD010216.

99.- RAHMAN M A, HANN N, WILSON A, MNATZAGANIAN G, WORRALL-CARTER L. E-cigarettes and smoking cessation: evidence from a systematic review and meta-analysis. PLoS One 2015; 10: $\mathrm{e} 0122544$.

100.- KALKHORAN S, GLANTZ S A. E-cigarettes and smoking cessation in real-world and clinical settings: a systematic review and meta-analysis. Lancet Respir Med 2016; 4: 116-28.

101.- HAJEK P, MCROBBIE H, MCROBBIE H, BULLEN
C. E-cigarettes and smoking cessation. Lancet Respir Med 2016; 4: e23.

102.- SIU A L, FOR THE U.S. PREVENTIVE SERVICES TASK FORCE. Behavioral and Pharmacotherapy Interventions for Tobacco Smoking Cessation in Adults, Including Pregnant Women: U.S. Preventive Services Task Force Recommendation Statement. Ann Intern Med 2015; 163: 622-34.

103.- BUCZKOWSKI K, MARCINOWICZ L, CZACHOWSKI S, PISZCZEK E. Motivations toward smoking cessation, reasons for relapse, and modes of quitting: results from a qualitative study among former and current smokers. Patient Prefer Adherence 2014; 8: 1353-63.

104.- CAPONNETTO P, POLOSA R, RUSSO C, LEOTTA C, CAMPAGNA D. Successful smoking cessation with electronic cigarettes in smokers with a documented history of recurring relapses: a case series. J Medical Case Reports 2011; 5: 585.

105.- CAHILL K, STEVENS S, LANCASTER T. Pharmacological treatments for smoking cessation. JAMA 2014; 311: 193-4

106.- BIENER L, HARGRAVES J L. A longitudinal study of electronic cigarette use among a population-based sample of adult smokers: association with smoking cessation and motivation to quit. Nicotine Tob Res 2015; 17: 127-33.

107.- HITCHMAN S C, BROSE L S, BROWN J, ROBSON D, MCNEILL A. Associations between e-cigarette type, frequency of use, and quitting smoking: findings from a longitudinal online panel survey in Great Britain. Nicotine Tob Res 2015; 17: 1187-94.

108.- PIERCE J P, GILPIN E A. Impact of over-the-counter sales on effectiveness of pharmaceutical aids for smoking cessation. JAMA 2002; 288: 1260-4.

109.- GLOBAL INITIATIVE FOR ASTHMA. Global Strategy for Asthma Management and Prevention, 2017. Disponible en: www.ginasthma.org

110.- GLOBAL INITIATIVE FOR CHRONIC OBSTRUCTIVE PULMONARY DISEASE. Global Strategy for the Diagnosis, Management, and Prevention of Chronic Obstructive Pulmonary Disease (2017 Report). Disponible en: www.goldcopd.org

111.- CHATKIN J M, ROCHA DULLIUS C. The management of asthmatic smokers. Asthma Research and Practice 2016; 2: 10.

112.- JIMÉNEZ-RUIZ C A, ANDREAS S, LEWIS K E, TONNESEN P, VAN SCHAYCK C P, HAJEK P, et al. Statement on smoking cessation in COPD and other pulmonary diseases and in smokers with comorbidities who find it difficult to quit. Eur Respir J 2015; 46: 6179.

113.- PERRET J L, BONEVSKI B, MCDONALD C F, ABRAMSON M J. Smoking cessation strategies for patients with asthma: improving patient outcomes. J Asthma Allergy 2016; 9: 117-28. 
114.- GRATZIOU C H, FLOROU A, ISCHAKI E, ELEFTHERIOU K, SACHLAS A, BERSIMIS S, et al.Smoking cessation effectiveness in smokers with COPD and asthma under real life conditions. Respir Med 2014; 108: 577-83.

115.- VOZORIS N T, STANBROOK M B. Smoking prevalence, behaviours, and cessation among individuals with COPD or asthma. Respir Med 2011; 105: 47784.

116.- TØNNESEN P. Smoking cessation and COPD. Eur Respir Rev 2013; 22: 37-43.

117.- TASHKIN D P. Smoking Cessation in Chronic Obstructive Pulmonary Disease. Semin Respir Crit Care Med 2015; 36: 491-507.

118.- DAUTZENBERG B, GARELIK D. Patients with lung cancer: are electronic cigarettes harmful or useful? Lung Cancer 2017; 105: 42-8.

119.- POLOSA R. Electronic cigarette use and harm reversal: emerging evidence in the lung. BMC Medicine 2015; 13: 54 .

120.- GONIEWICZ M, GAWRON M, SMITH D M, PENG M, JACOB III P, BENOWITZ N. Exposure to nicotine and selected toxicants in cigarette smokers who switched to electronic cigarettes: a longitudinal within subjects observational study. Nicotine Tob Res 2016; 19: 160-7.

121.- POLOSA R, MORJARIA J B, CAPONNETTO P, CARUSO M, CAMPAGNA D, AMARADIO M D et al. Persisting long term benefits of smoking abstinence and reduction in asthmatic smokers who have switched to electronic cigarettes. Discov Med 2016; 21: 99-108.

122.- POLOSA R, MORJARIA J B, CAPONNETTO P, PROSPERINI U, RUSSO C, PENNISI A et al. Evidence for harm reduction in COPD smokers who switch to electronic cigarettes. Respir Research 2016; 17: 166 doi: 10.1186/s12931-016-0481-x.

123.- LUCCHIARI C, MASIERO M, VERONESI G, MAISONNEUVE P, SPINA S, JEMOS C et al. Benefits of E-Cigarettes Among Heavy Smokers Undergoing a Lung Cancer Screening Program: Randomized
Controlled Trial Protocol. JMIR Res Protoc 2016; 5: e21.

124.- SUTER M A, MASTROBATTISTA J, SACHS M, AAGAARDIS K. There Evidence for Potential Harm of Electronic Cigarette Use in Pregnancy? Birth Defects Res A Clin Mol Teratol 2015; 103: 186-95.

125.- GIBBS K, COLLACO J M, MCGRATH-MORROW S A. Impact of Tobacco Smoke and Nicotine Exposure on Lung Development. Chest 2016; 149: 552-61.

126.- COLEMAN T, CHAMBERLAIN C, COOPER S, LEONARDI-BEE J. Efficacy and safety of nicotine replacement therapy for smoking cessation in pregnancy: systematic review and meta-analysis. Addiction 2011; 106: 52-61.

127.- COLEMAN T, COOPER S, THORNTON J G, GRAINGE M J, WATTS K, BRITTON J et al. Smoking, Nicotine, and Pregnancy (SNAP) Trial Team. A randomized trial of nicotine-replacement therapy patches in pregnancy. N Engl J Med 2012; 366: 808-18.

128.- HOLBROOK B D. The effects of nicotine on human fetal development. Birth Defects Res C Embryo Today 2016; 108: 181-92.

129.- GONIEWICZ M L, LEE L. Electronic Cigarettes Are a Source of Thirdhand Exposure to Nicotine. Nicotine Tob Res 2014; 17: 256-8.

130.- MIDDLEKAUF H R. Counterpoint: Does the Risk of Electronic Cigarettes Exceed Potential Benefi ts? No.Chest 2015; 148: 582-4.

131.- CHEN L S, HORTON A, BIERUT L. Pathways to precision medicine in smoking cessation treatments. Neuroscience Letters. http://dx.doi.org/10.1016/j.neulet.2016.05.033

132.- SONEJI J, BARRINGTON-TRIMIS J L, WILLS T A, LEVENTHAL A M, UNGER J B, GIBSON L A et al. Association between initial use of e-cigarettes and subsequent cigarette smoking among adolescents and young adults. JAMA Pediatrics 2017. Doi: 10.1001/jamapediatrics.2017.1488 Disponible en: http://jamanetwork.com/journals/jamapediatrics/fullarticle/2634377 (consultada el 19 de julio de 2017).

Correspondencia a:

Dr. Gonzalo Alvear T.

Grupo Respiratorio Integramédica

Américo Vespucio 1501.

Comuna de Cerrillos.

Región Metropolitana, Chile.

Email: galveart@hotmail.com 